\title{
Quantitative factors proposed to influence the prevalence of canine tick-borne disease agents in the United States
}

\author{
Roger W Stich ${ }^{1 *}$, Byron L Blagburn ${ }^{2}$, Dwight D Bowman ${ }^{3}$, Christopher Carpenter $^{4}$, M Roberto Cortinas $^{5}$, \\ Sidney A Ewing ${ }^{6}$, Desmond Foley ${ }^{7}$, Janet E Foley ${ }^{8}$, Holly Gaff ${ }^{9}$, Graham J Hickling ${ }^{10}$, R Ryan Lash ${ }^{11}$, Susan E Little ${ }^{6}$, \\ Catherine Lund ${ }^{12}$, Robert Lund ${ }^{13}$, Thomas N Mather ${ }^{14}$, Glen R Needham ${ }^{15}$, William L Nicholson ${ }^{16}$, Julia Sharp ${ }^{13}$, \\ Andrea Varela-Stokes ${ }^{17}$ and Dongmei Wang ${ }^{13}$
}

\begin{abstract}
The Companion Animal Parasite Council hosted a meeting to identify quantifiable factors that can influence the prevalence of tick-borne disease agents among dogs in North America. This report summarizes the approach used and the factors identified for further analysis with mathematical models of canine exposure to tick-borne pathogens.
\end{abstract}

Keywords: Anaplasma, Ehrlichia, Borrelia burgdorferi, Tick-borne infections, Prevalence map factors, Ticks, Ixodidae, Prostriata, Metastriata

\section{Background}

Dogs in the United States (USA) are hosts to a diverse range of ixodid ticks and can become infected with many of the pathogens transmitted by these vectors. Advances in diagnostic test and recording technologies have led to the creation of a monthly dataset containing county-by-county canine test results from across the USA. The Companion Animal Parasite Council (CAPC) has assembled large datasets of such results from commercial laboratories that provide diagnostic tests for canine exposure to Borrelia burgdorferi, Ehrlichia spp. and Anaplasma spp. [1]. These monthly, county-level CAPC prevalence maps generated interest in the utility of the datasets for assessing seroprevalence norms, forecasting future seroprevalence rates and for identifying trends in canine exposure to this array of tick-borne disease agents. A group of vector ecologists, parasitologists, other biologists and statistical modelers met in Atlanta, GA (June 9-10, 2012) to identify factors that could enhance the accuracy of these predictive models. This report narrates the results of the meeting.

\footnotetext{
* Correspondence: stichrw@missouri.edu

${ }^{1}$ Department of Veterinary Pathobiology, University of Missouri, Columbia, $\mathrm{MO}$, USA

Full list of author information is available at the end of the article
}

Canine diagnostic test results for exposure to tickborne pathogens, including B. burgdorferi, Ehrlichia spp. and Anaplasma spp., are of significant interest, not only because canine health is important to pet owners and veterinarians, but also because of the public health importance of many of these infectious disease agents. These tick-borne pathogens are transmitted by two phylogenetically distinct groups of ixodid ticks. Members of the ixodid subfamily Prostriata (Ixodes spp.) transmit agents of granulocytic anaplasmosis (Anaplasma phagocytophilum) and Lyme borreliosis (B. burgdoferi) and are likely to include vectors of a more recently described Ehrlichia muris-like agent in the USA. Members of the subfamily Metastriata (e.g., the genera Amblyomma, Dermacentor and Rhipicephalus) transmit agents of canine and human ehrlichiosis (e.g., E. canis, E. chaffeensis and $E$. ewingii), canine anaplasmosis (A. platys) and spotted-fever group rickettsiosis (i.e., Rickettsia rickettsii, R. conorii and related Rickettsia spp.).

Large datasets have been assembled from reports of diagnostic test results for canine exposure to B. burgdorferi, Anaplasma spp. and Ehrlichia spp. in the USA. For example, from reports submitted nationwide from 2010-2012, 509,195 (7.2\%) of 6,996,197 canine samples were seropositive for B. burgdorferi, 270,168 (4.4\%) of 6,192,268 samples were seropositive for Anaplasma, 
and $111,673(1.1 \%)$ of $6,994,683$ samples were seropositive for Ehrlichia [2]. A previous national survey, spanning 2001-2007, reported results from 982,336 diagnostic tests for canine exposure to B. burgdorferi and Ehrlichia spp., and 479,640 tests for canine antibodies to Anaplasma spp., with $5.1 \%, 0.6 \%$ and $4.7 \%$ of these samples testing seropositive for B. burgdorferi, Ehrlichia and Anaplasma, respectively [3]. Interestingly, when the canine seroprevalence of $B$. burgdorferi in the 2001-2007 study was compared to the subsequent prevalence of human Lyme disease, the most commonly reported human vector-borne illness in the USA, canine seroprevalence of B. burgdorferi $\geq 5.1 \%$ was predictive of emergent human Lyme disease in low-incidence counties; a low canine seroprevalence $(\leq 1.0 \%)$ was associated with minimal risk for emergent human Lyme disease [4]. A subsequent report, however, underscored the importance of other variables, such as the distribution of competent vector species, for accurate interpretation of these canine diagnostic test data [5].

The overall objective of this CAPC-sponsored workshop was to identify factors that are likely to influence the seroprevalence of canine exposure to tick-borne disease agents in the USA, specifically focusing on the factors and the pathogens for which sufficient data are available, so that these factors could be evaluated for incorporation in mathematical models designed to monitor and to predict spatial and temporal seroprevalence patterns. These preliminary factors provided statisticians some of the critical information needed to begin their model-building procedures.

\section{Approach}

Two teams of researchers, from various areas of tick and tick-borne pathogen biology, were assembled and tasked with rational identification of factors thought to be relevant to the canine seroprevalence of pathogens transmitted by prostriate (eight team members) or metastriate (seven team members) ticks (Figure 1). Members of each team were selected based upon diverse areas of expertise in tick biology, tick-borne disease, vector ecology or statistics. Each panel was asked to identify and to rank ten key factors that they considered most likely to affect pathogen seroprevalence, and these preliminary factors were then presented to all of the meeting participants for further discussion. It was understood that the relevance of these factors would be subsequently assessed with mathematical models, and that these models would be adjusted with data that continue to be generated. Thus, the utility of different factors would be continually assessed as the mathematical models are refined over time.
The working groups for both ixodid subfamilies began by discussing variables categorized as (1) vector, (2) host, (3) abiotic, (4) habitat or (5) social. Both groups independently identified numerous factors. The majority of factors were thought to be associated with canine exposure to pathogens vectored by either ixodid subfamily; however, several factors specifically associated with the different ixodid subfamilies also emerged. Variables were also discussed for which there is little or inconsistent supporting data, but these factors could become useful if the data became available. However, in accordance with the workshop objectives, factors for which sufficient data are currently available were chosen for ranking by consensus of each working group.

The variables independently identified by each panel were categorized into the five groups previously indicated (i.e., vector, host, abiotic, habitat and social). Factors regarding exposure to infectious agents transmitted by prostriate ticks were heavily influenced by the preponderance of research on the phenology of Ixodes scapularis and I. pacificus, which are considered the primary vectors of $B$. burgdorferi and A. phagocytophilum in North America (Table 1). For metastriate-borne pathogens, host biology and human behavior were second only to vector distribution with regard to factors considered likely to influence seroprevalence (Table 2). Brief explanations and comments regarding these factors are described below.

\section{Vector factors \\ Distribution}

The geographic distribution of prostriate ticks was focused on the Ixodes spp. thought to most commonly feed on dogs (and people) in the USA: I. scapularis and I. pacificus. Metastriate ticks considered as pathogen vectors (e.g., of Ehrlichia spp. and A. platys) included, in alphabetical order, A. americanum, A. maculatum, D. andersoni, D. variabilis and $R$. sanguineus. The general distributions of these ticks are relatively well documented in the literature and via voucher specimens in the USA. However, the spatial resolutions of these data vary in different regions, and defining the minimum useful scale can be complicated by discontinuous geographic distributions of tick populations in a given area.

\section{Abundance}

Defining permanent values of tick abundance levels is problematic, because tick population levels within a given area are temporally and spatially variable and can change rapidly. Tick abundance depends on host abundance and availability, relative humidity, precipitation and temperature, and can reflect conditions from previous years when immature tick stages or prior generations were active. 


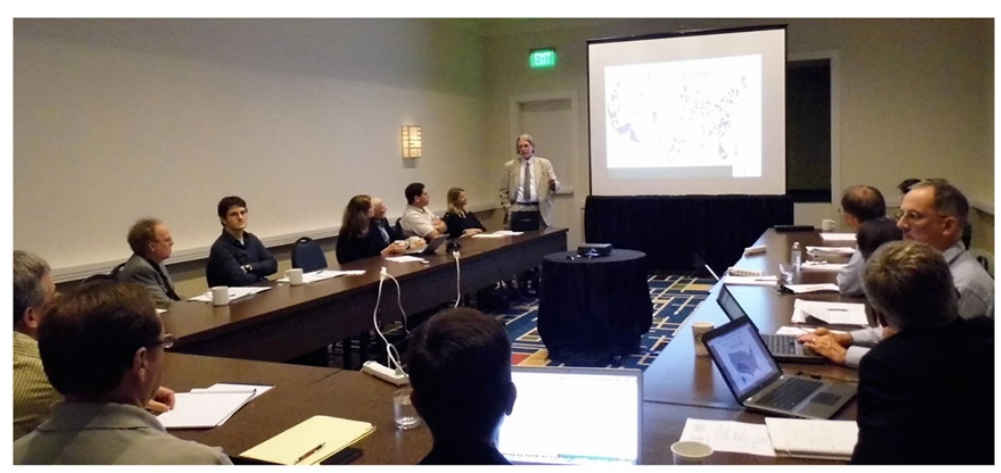

Background and objectives described to meeting participants

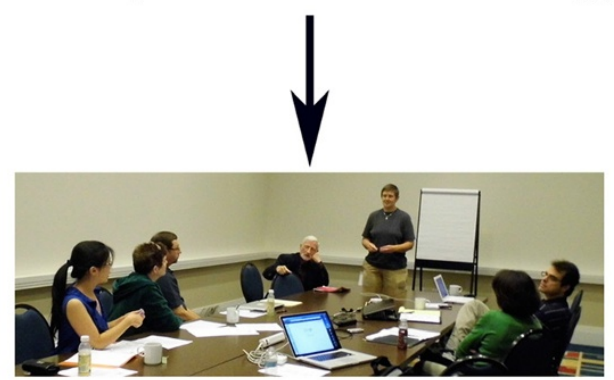

Participants divided into panels to identify prospective factors in accordance with the criteria provided

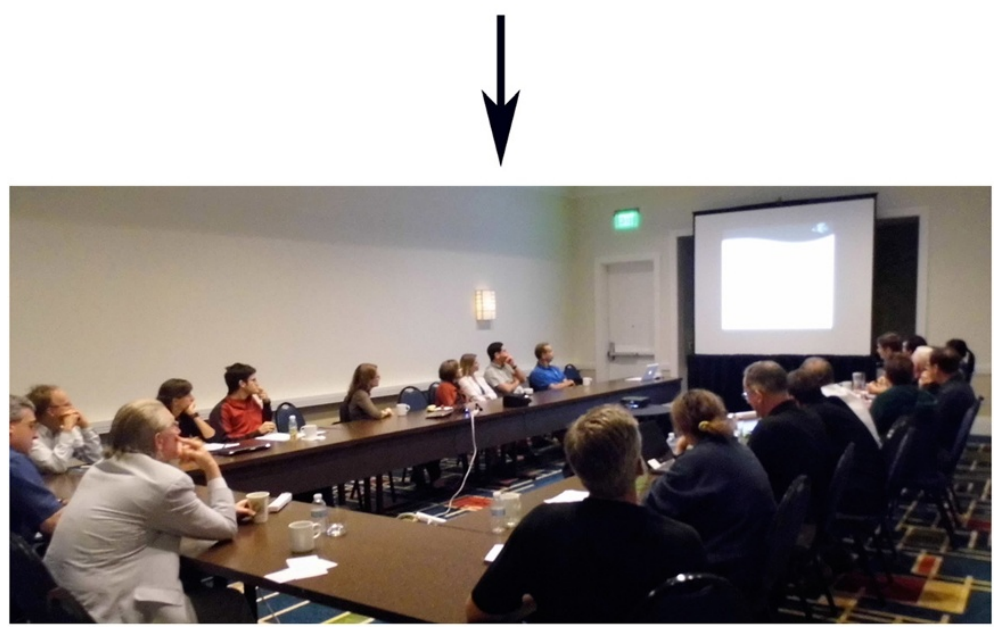

Participants reconvened to discuss the results of each panel

Figure 1 Approach to rational identification of quantitative factors proposed to influence the exposure of dogs to vector-borne pathogens. Background information, meeting objectives and guidelines were presented to participants before they were divided into three separate panels, according to vector taxa, for mosquitoes, prostriate ticks and metastriate ticks. Each panel was asked to identify, discuss and to rank candidate factors for evaluation with statistical prevalence models of pathogens transmitted to US dogs by each vector taxon. All of the participants were subsequently reconvened for further discussion and refinement of the results from each panel.

\section{Activity}

Activity is indicative of questing behavior, host-seeking behavior, host contact and the feeding preferences of different developmental stages. The presence of ticks in an area is not alone indicative of activity. For example, tick activity will depend on temperature, precipitation, relative humidity and photoperiod.

\section{Host factors}

Deer

The deer population is a major driver of abundance for certain ticks, such as I. scapularis, I. pacificus and A. americanum. Deer are also a reservoir of $E$. chaffeensis and could be involved in the maintenance of E. ewingii. 


\section{Table 1 Factors initially considered as potential contributors to canine prevalence of disease agents transmitted by Ixodes scapularis and I. pacificus}

\begin{tabular}{l}
\hline Vector factors \\
\hline Distribution \\
Abundance \\
\% Infected \\
Canine contact \\
Local phenology \\
Tolerance to temperature and humidity \\
Activity \\
Focus on adults as primary vector to dogs \\
Host seeking behavior \\
Host contact \\
Feeding preferences and opportunities \\
Deer population drives tick abundance \\
Small mammal population drives infection prevalence \\
Lack of lizards \\
Diversity/dilution effect \\
Tick encounters \\
Questing behavior versus relative humidity \\
Peridomestic encounters - access to areas \\
Urbanization/Rate of development \\
Infection status (decreased survival versus increased cold \\
Holerance)
\end{tabular}

\section{Host factors}

Presence and abundance (deer, small mammals, lizards)

Dilution effect/host diversity

Habitat availability and quality

Mast crop as a surrogate for host reproduction/fitness

Migratory bird patterns

Reproductive capacity and timing of vertebrate host reproduction

Population control programs in place locally

Abiotic host survival factors

Temperature, water availability, substrate/nesting material, snow cover

Feeding preferences

Herd immunity of reservoir host populations

Hunting pressure/success

Number of deer killed per county - harvest rates

Hunting license versus hunting harvest - how active hunting is for that area

Hunting limits due to development

\section{Abiotic factors}

Snow cover - depth, duration

Miles of roads - neighborhood roads (non-interstate/parkway/ highway), trails

Soil type - clay versus sand in Northeastern USA
Table 1 Factors initially considered as potential contributors to canine prevalence of disease agents transmitted by Ixodes scapularis and I. pacificus (Continued)

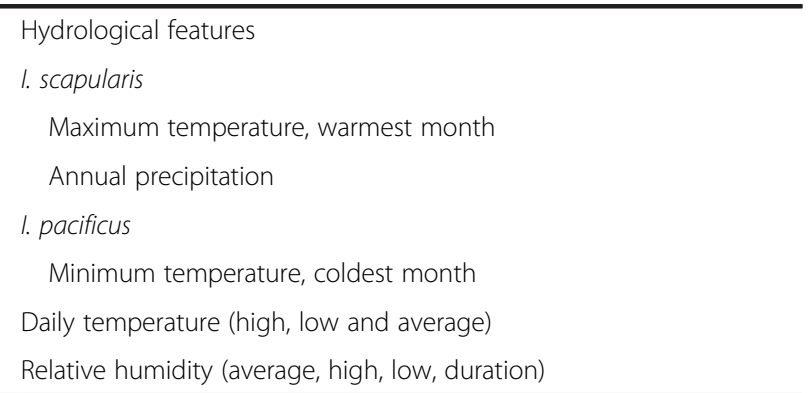

\section{Habitat factors}

Land cover classification

Urbanization in 3 categories - low, medium, high

Rate of change

Forest cover

Land cover classification (categorical), \% canopy cover, NDVI, EVI (canopy structure)

Crop phenology - maximum greening, minimum greening when greening is happening

Supervised vs unsupervised satellite imagery, derived data not currently off the shelf

Forest type, forest fragmentation, forest edge length, forest composition, forest connectivity

Forest fragments within $\mathrm{X}$ distance of road or urban area, close to population centers

Understory- could be modeled but is not measured

Detritus layers/leaf litter

Targeted for future research but perhaps not currently available dataset

Soil maps/soil types

World harmonized soil database

Classification scheme

Proximity to rivers/drainage areas

Proximity to coast

Rain shadows

Rivers and streams

\section{Attract hosts}

Serve as corridors

Provide humidity

Aspect/slope/topo index - derived from digital elevation models, available from hydro dataset

More nymphal deer ticks on north- and east-facing slopes

Effective distance - more ticks on uphill side of a payout

Ticks associated with east-facing woodland edges that slope down to water

Fire

Eliminates leaf litter, changes food availability, changes microclimate 


\section{Table 1 Factors initially considered as potential contributors to canine prevalence of disease agents transmitted by Ixodes scapularis and $I$. pacificus (Continued)}

Depending on timing, burn can increase number of infected ticks, so fewer ticks but higher infection rate

Park boundaries - proximity to parks

Social factors
Human population centers
Dog ownership, dog lifestyle
Hunting styles that use dogs
Breed of dog
Dog ownership increase - by region
More homes in tick habitat - demographic factors
Deer/vehicle collisions - deer crossing signs
Acaricide use/quality of care for dogs
Average household income
Presence of clinics, proximity to clinics, number of vet clinics in an
area, size of clinics
Cultural - forest foraging (mushroom hunting in Missouri)
Internet use
Social media
Smartphone use
Education level
Population density
Housing type (average lot size, median home price, age of house
unit, census tract size)

\section{Small mammals}

Rodents are an important component of the ecologies of several tick species and some tick-borne infectious agents. Immature stages of several tick species acquire blood meals from small vertebrate hosts. Several tickborne infectious agents, such as B. burgdorferi, $A$. phagocytophilum and $R$. rickettsii are adapted to rodent reservoir hosts.

\section{Lizards}

Small vertebrates such as lizards, which are permissive hosts for immature tick stages but are not definitively documented reservoirs of the pathogens under consideration, could dampen transmission of disease agents that are adapted to rodent reservoirs. Conversely, removal of lizards reportedly reduced nymphal tick numbers from an environment but did not affect the percentage of $B$. burgdorferi-positive ticks, suggesting that increased numbers of lizard hosts might actually increase the risk of pathogen transmission by serving to increase the overall number of ticks in a given area [6].

\section{Migratory bird patterns}

Migratory birds can introduce some tick species to new areas [7]. However, ticks that feed on dogs and that are dispersed by birds in the USA may be incapable of maintaining an active population cycle in the absence of larger vertebrate hosts (e.g., white-tailed deer).

\section{Abiotic factors}

Different tick species and their natural hosts can be adapted to various environments that are influenced by abiotic factors such as precipitation, temperature, relative humidity and soil composition.

\section{Habitat factors}

Factors that influence the life cycles of ticks and their vertebrate hosts include vegetation, urbanization, land use in non-urban settings and detritus layers.

\section{Social factors}

Human behavior and population characteristics influence the exposure of dogs to ticks. These include access to preventive care, recreation, socioeconomic status, income, pathogen reservoir control, vector-amplification host control and news media coverage.

\section{Unquantified variables}

A number of variables were discussed for which comprehensive, nationwide data did not seem currently available. These variables included vector infection rates, detailed reservoir infection rates, vector abundances, vector efficiency indices, vector survival, vectorial capacities, temperature-dependent development rates of vectors (natural temperature regimes), total number of dogs (by county or zip code) and tick control product sales in each geographic region. Local data may be available for some of these variables in certain areas, but national datasets were not available at the time of this meeting.

\section{Mathematical modeling}

Each expert panel was asked to prioritize 10 factors most expected to drive a reliable mathematical predictive model. These lists, summarized in Tables 3 and 4, shared several common abiotic and habitat factors. Several other factors were specific to seroprevalence of the pathogens transmitted by Ixodes spp., $R$. sanguineus or the other metastriate ticks that were considered. For example, while deer populations and vegetation were considered important factors that affect the majority of these tick populations, social factors were given the highest priority for predicting the seroprevalence of agents transmitted by the brown dog tick, $R$. sanguineus (Table 4). 
Table 2 Factors discussed as potential contributors to seroprevalence of metastriate tick-borne pathogens among dogs in the USA

\begin{tabular}{ll}
\hline Vector factors & Biology \\
Competence (different transmission scenarios) \\
Host preference \\
Persistence and interhost transfer of male ticks \\
Host seeking behavior (hunt, ambush) \\
Population dynamics \\
Distribution (established, intermittent or absent) \\
Relative abundance (species and stages) \\
Seasonality \\
Different stages \\
Stage overlap
\end{tabular}

Host factors

Principal host(s) of different tick stages

Susceptibility to pathogen

Distribution

Density-Dynamic

Ecologic diversity (dilution effect)

Shannon-Weaver Index

Tick-permissive, non-reservoir hosts

Behavior

Host grooming

Gregariousness

Host species

Home Range

Migration, dispersal

Anthropogenic translocation

Hosts permissive for pathogen

Persistence in reservoir

Prevalence of infection

Density

Other transmission routes

Life cycle/age distribution

Immune response

Amplification vs. reservoir

Domestic

Indoor/outdoor

Rural/urban

Relocation

Sylvatic vs. Suburban

Opportunistic or natural infection

Abiotic factors

Humidity

Maximum, minimum and average
Table 2 Factors discussed as potential contributors to seroprevalence of metastriate tick-borne pathogens among dogs in the USA (Continued)

\begin{tabular}{|c|c|}
\hline & Temperature \\
\hline & Maximum, minimum and average \\
\hline & Degree-day \\
\hline & Soil temperature \\
\hline & Photoperiod \\
\hline & Seasonal precipitation \\
\hline & El Niño effect \\
\hline & Snow and other ground cover \\
\hline & Catastrophic disturbance \\
\hline & Fire \\
\hline & Hurricane \\
\hline & Wind \\
\hline & Altitude \\
\hline Habitat factors & \\
\hline & Macrohabitat \\
\hline & Vegetation (density, type and fragmentation) \\
\hline & Elevation \\
\hline & Location of water sources \\
\hline & Rainfall \\
\hline & Microhabitat \\
\hline & Soil type \\
\hline & LIDAR data \\
\hline & Land use \\
\hline Social factors & \\
\hline & Land use \\
\hline & Indoor versus outdoor dogs \\
\hline & Dog use (e.g., hunting) \\
\hline & Canine husbandry \\
\hline & Use of tick preventives \\
\hline & Nuisance permits \\
\hline & Housekeeping \\
\hline & Animal welfare violations \\
\hline & Socioeconomics \\
\hline & Average household income \\
\hline & Human population \\
\hline & Large-scale economic factors \\
\hline & Recreation \\
\hline & Hunting \\
\hline & Parks (rural and urban) \\
\hline & Pets per household \\
\hline
\end{tabular}


Table 3 Ranked factors identified for canine seroprevalence models of infections transmitted by Ixodes spp. in the USA

1. ${\text { Forest cover/NDVI or } E V l^{a}}^{\text {2. }}$ Relative humidity
3. Annual precipitation (including snow cover) ${ }^{\mathrm{a}}$
4. Human population density ${ }^{\mathrm{a}}$
5. Deer/vehicle collisions ${ }^{\mathrm{a}}$
6. Topography/altitude/aspect
6. Temperature - max warmest, min coldest ${ }^{\mathrm{a}}$
7. Proximity of forest to impervious surfaces or roads/built
8. Human case distribution
8. Distribution/abundance of I. scapularis and I. pacificus ${ }^{\mathrm{a}}$
9. Household income
10. Forest fragmentation index

${ }^{\mathrm{a}}$ Similar variables also ranked by the metastriate-borne pathogen panel.

The prevalence data at the foundation of this predictive model is largely based on serodiagnostic tests. Although seropositivity is reflective of past exposure, it does not demonstrate recent or active infections. Repeatedly seropositive samples from the same dogs at different times are also to be occasionally expected, because some dogs may have tested seropositive in previous tests and because some tests are conducted to monitor host responses to treatment. Travel histories and certainties of the individual test results are currently unavailable for the dogs reported in this dataset.

An analogous project for mathematical modeling of the prevalence of canine heartworm was simultaneously undertaken by CAPC $[8,9]$, and each prioritized factor identified by the expert panel had significant predictive power with $\geq 95 \%$ confidence. Overall, the model explained $60 \%-70 \%$ of variability in the CAPC county-by-county dataset from 2011-2013. Similarly, preliminary analysis of canine seroprevalence of Anaplasma spp. indicated that temperature, precipitation, relative humidity, population density, median household income, forestation coverage, elevation and deer/vehicle strike rates were significant with $\geq 95 \%$ confidence, and that the total proportion of variability explained in the 2011-2013 data is around $60-70 \%$ [10]. Thus, the prevalence of heartworm and seroprevelance of Anaplasma among dogs appear amenable to quantification that could facilitate monitoring for outbreaks, remediation of vector abundance or for forecasting future seroprevalence levels.

Attempts to fit the seroprevalence of B. burgdorferi and of Ehrlichia spp. among dogs are also underway, with mixed results. The spatial seroprevalence of $B$. burgdorferi among dogs has been similar to and appears to be as quantifiable as that of Anaplasma spp. Conversely,
Table 4 Ranked factors for preliminary models of metastriate tick-borne pathogen prevalence among dogs in the USA

\begin{tabular}{|c|c|}
\hline \multicolumn{2}{|c|}{ Majority of the metastriata: } \\
\hline 1. & Vector distribution (established, intermittent or absent) ${ }^{\mathrm{a}}$ \\
\hline 2. & Maximum, minimum and average temperature ${ }^{b}$ \\
\hline 3. & Amount of precipitation ${ }^{a}$ \\
\hline 4. & LiDAR (up to 6 layers) \\
\hline 5. & GAP/categorical analysis of vegetation ${ }^{a}$ \\
\hline 6. & Reservoir host densities ${ }^{a}$ \\
\hline 7. & Human population (census) $)^{a, b}$ \\
\hline 8. & Median household income $e^{a, b}$ \\
\hline 9. & Fragmentation of vegetation ${ }^{\mathrm{b}}$ \\
\hline 10. & Degree-days \\
\hline 11. & Seasonal precipitation (snow cover) ${ }^{a}$ \\
\hline \multicolumn{2}{|c|}{ R. sanguineus: } \\
\hline 1. & Median household income ${ }^{a, b}$ \\
\hline 2. & Registered dog breeders (kennels, puppy mills, etc.) \\
\hline 3. & Human population (census) $)^{a, b}$ \\
\hline 4. & Tick preventive sales \\
\hline 5. & Animal welfare violations \\
\hline 6. & Latitude \\
\hline
\end{tabular}

${ }^{a}$ Variables also ranked by the prostriate-borne pathogen panel.

bariables shared among all ixodid ticks considered for this report.

the canine seroprevalence of Ehrlichia spp. appears to be highly variable, with some neighboring areas reporting antipodal seroprevalence rates that could be reflective of vector ecology or social factors. Future work will address these issues.

\section{Conclusions}

This meeting brought together a range of junior and senior scientists engaged in various aspects of research in the biology of ticks and tick-borne infections. The specific objectives were to identify and to prioritize quantifiable factors expected to contribute to canine exposure to organisms transmitted by the two major subfamilies of ixodid ticks. The two panels ranked 12 and 17 factors associated with prostriate and metastriate ixodid ticks, respectively. Eight of these factors were independently prioritized by both panels; four of 12 factors were unique to prostriatevectored agents, two of 11 factors were unique to metastriate-vectored agents transmitted by ticks other than $R$. sanguineus, and four of six factors were unique to agents vectored by $R$. sanguineus. The next phase of this project will move from rational identification of perceived factors to statistical assessment of factors for predictive power. Forecasting issues will also be explored. 


\section{Competing interests}

The authors have no competing interests relative to the work presented in this report.

\section{Authors' contributions}

SEL, DDB, CL, CC, RL and RWS conceived of and organized the meeting that generated the information provided in this report. BLB, DDB, CC, MRC, SAE, DF, JEF, HG, GJH, RRL, SEL, CL, RL, TM, GRN, WLN, JS, RWS, AV-S and DW participated in the meeting and contributed to the identification of candidate factors. RWS tabulated the candidate factors from the meeting minutes, and SEL, DDB and RWS generated the initial draft of the manuscript. RWS was responsible for distribution of the manuscript to the co-authors, for revision in response to comments, and for formatting the final manuscript version for submission. All authors read and approved the final version of the manuscript.

\section{Acknowledgments}

This meeting was supported by the Companion Animal Parasite Council (CAPC), and we are grateful to Sonya Hennessy for assistance in organizing and hosting this meeting. The CAPC is in turn grateful to its sponsors that provide data for Parasite Prevalence Maps: the IDEXX, Antech, Banfield and Abaxis corporations. We are also grateful to the veterinarians across the USA who test their patients for exposure to vector-borne pathogens, especially those who report test results that can eventually be included as data in the CAPC Parasite Prevalence Maps. Robert Lund acknowledges support from the CAPC and from the National Science Foundation Grant DMS-1407480. The opinions and assertions contained herein are those of the authors and are not to be construed as official or reflecting the views of the Department of the Army or the Department of Defense.

\section{Author details}

${ }^{1}$ Department of Veterinary Pathobiology, University of Missouri, Columbia, MO, USA. Department of Pathobiology, Auburn University, Auburn, AL, USA. ${ }^{3}$ College of Veterinary Medicine, Cornell University, Ithaca, NY, USA.

${ }^{4}$ Companion Animal Parasite Council, Salem, OR, USA. ${ }^{5}$ School of Veterinary Medicine and Biomedical Sciences, University of Nebraska, Lincoln, NE, USA. ${ }^{6}$ Department of Veterinary Pathobiology, Oklahoma State University, Stillwater, OK, USA. ${ }^{7}$ Walter Reed Biosystematics Unit, National Museum of Natural History, Washington, DC, USA. ${ }^{8}$ Department of Medicine and Epidemiology, University of California, Davis, CA, USA. ${ }^{9}$ Department of Biological Sciences, Old Dominion University, Norfolk, VA, USA. ${ }^{10}$ Department of Forestry, Wildlife and Fisheries, University of Tennessee, Knoxville, TN, USA. ${ }^{11}$ Department of Geography, University of Georgia, Athens, GA, USA. ${ }^{12}$ City Kitty Veterinary Care for Cats, Providence, RI, USA. ${ }^{13}$ Department of Mathematical Sciences, Clemson University, Clemson, SC, USA. ${ }^{14}$ Center for Vector-Borne Disease, University of Rhode Island, Kingston, RI, USA.

${ }^{15}$ Department of Entomology, The Ohio State University, Columbus, OH, USA. ${ }^{16}$ Centers for Disease Control and Prevention, Atlanta, GA, USA.

${ }^{17}$ Department of Basic Sciences, College of Veterinary Medicine, Mississippi State University, Mississippi State, MS, USA.

Received: 19 August 2014 Accepted: 30 August 2014 Published: 4 September 2014

\section{References}

1. Companion Animal Parasite Council Parasite Prevalence Maps. [http://www.capcvet.org/parasite-prevalence-maps]

2. Little SE, Beall MJ, Bowman DD, Chandrashekar R, Stamaris J: Canine infection with Dirofilaria immitis, Borrelia burgdorferi, Anaplasma spp., and Ehrlichia spp. in the United States, 2010-2012. Parasit Vectors 2014, 7(1):257.

3. Bowman D, Little SE, Lorentzen L, Shields J, Sullivan MP, Carlin EP: Prevalence and geographic distribution of Dirofilaria immitis, Borrelia burgdorferi, Ehrlichia canis, and Anaplasma phagocytophilum in dogs in the United States: results of a national clinic-based serologic survey. Vet Parasitol 2009, 160(1-2):138-148.

4. Mead P, Goel R, Kugeler K: Canine serology as adjunct to human Lyme disease surveillance. Emerg Infect Dis 2011, 17(9):1710-1712.

5. Millen K, Kugeler KJ, Hinckley AF, Lawaczeck EW, Mead PS: Elevated lyme disease seroprevalence among dogs in a nonendemic county: harbinger or artifact? Vector Borne Zoonotic Dis 2013, 13(5):340-341.

6. Swei A, Ostfeld RS, Lane RS, Briggs CJ: Impact of the experimental removal of lizards on Lyme disease risk. Proc Biol Sci 2011, 278(1720):2970-2978.
7. Elias SP, Smith RP Jr, Morris SR, Rand PW, Lubelczyk C, Lacombe EH: Density of Ixodes scapularis ticks on Monhegan Island after complete deer removal: a question of avian importation? J Vector Ecol 2011, 36(1):11-23.

8. Wang D, Bowman DD, Brown HE, Harrington LC, Kaufman PE, McKay T, Nelson CT, Sharp JL, Lund R: Factors influencing U.S. canine heartworm (Dirofilaria immitis) prevalence. Parasit Vectors 2014, 7(1):264.

9. Brown HE, Harrington LC, Kaufman PE, McKay T, Bowman DD, Nelson CT, Wang D, Lund R: Key factors influencing canine heartworm, Dirofilaria immitis, in the United States. Parasit Vectors 2012, 5:245.

10. Wang D, Bowman DD, Goldstein R, McMahan CS, Sharp JL, Beal MJ, Stich RW, Lund R: Factors Associated with the Prevalence of Canine Exposure to Anaplasma spp. in the United States. Unpublished observations.

\section{doi:10.1186/1756-3305-7-417}

Cite this article as: Stich et al:: Quantitative factors proposed to influence the prevalence of canine tick-borne disease agents in the United States. Parasites \& Vectors 2014 7:417.

\section{Submit your next manuscript to BioMed Central and take full advantage of:}

- Convenient online submission

- Thorough peer review

- No space constraints or color figure charges

- Immediate publication on acceptance

- Inclusion in PubMed, CAS, Scopus and Google Scholar

- Research which is freely available for redistribution

Submit your manuscript at www.biomedcentral.com/submit
C) Biomed Central 